Relations industrielles

Industrial Relations

\title{
The Israeli Worker, by Ferdynand Zweig, Herzl Press and Sharon Books, New-York, 1959, 305 pp., \$5.00.
}

\section{Gérald Fortin}

Volume 16, numéro 2, avril 1961

URI : https://id.erudit.org/iderudit/1021822ar

DOI : https://doi.org/10.7202/1021822ar

Aller au sommaire du numéro

Éditeur(s)

Département des relations industrielles de l’Université Laval

ISSN

0034-379X (imprimé)

1703-8138 (numérique)

Découvrir la revue

Citer ce compte rendu

Fortin, G. (1961). Compte rendu de [The Israeli Worker, by Ferdynand Zweig, Herzl Press and Sharon Books, New-York, 1959, 305 pp., \$5.00.] Relations industrielles / Industrial Relations, 16(2), 276-276.

https://doi.org/10.7202/1021822ar

Tous droits réservés (C Département des relations industrielles de l’Université Laval, 1961
Ce document est protégé par la loi sur le droit d'auteur. L’utilisation des services d'Érudit (y compris la reproduction) est assujettie à sa politique d'utilisation que vous pouvez consulter en ligne.

https://apropos.erudit.org/fr/usagers/politique-dutilisation/ 
où le développement sanitaire du pays se trouve dangereusement freiné par le manque croissant de personnel qualifié pour les soins, une telle étude ne se contente pas d'être un cri d'alarme, elle propose aussi des éléments de solution.

\section{L.}

The Israeli Worker, by Ferdynand Zweig, Herzl Press and Sharon Books, New York, 1959, 305 pp., \$5.00.

L'auteur, avant de visiter Israël, croyait $\mathbf{y}$ trouver les mêmes genres de relations de travail et d'attitudes que dans les autres pays de l'Occident. Au contraire, nous dit-il, en Israël, tout est neuf, étrange, unique. Autant le paysage est riche et varié, autant le monde ouvrier est disparate et mouvant. Chaque groupe de travailleurs a ses problèmes particuliers de même que sa façon originale d'y apporter des solutions. Il est d'ailleurs d'autant plus difficile d'étudier ce monde du travail, qu'il est à peine naissant. Il n'existe aucune tradition dans ce domaine. Tout est à faire et des solutions nouvelles sont inventées à chaque jour.

L'état d'Israël doit trouver des moyens rapides pour assurer la prospérité du pays. L'industrialisation semble une des voies les plus sûres, mais elle suppose une expérience que ne possède pas la population. Une faible minorité des immigrants juifs sont des travailleurs manuels. Les ouvriers israéliens sont d'anciens collets blancs pour la plupart. Non seulement doivent-ils apprendre de nouvelles techniques, mais ils doivent aussi apprendre à devenir une nouvelle classe sociale. Ils doivent apprendre à avoir d'eux-mêmes une nouvelle image et à accepter cette nouvelle image.

Dans une société où s'affrontent le traditionalisme judaïque et un socialisme radical, une population autochtone et un flot constant d'immigrants, où le chômage est élevé et la sécurité d'em- ploi longue à obtenir, des conflits sont inévitables. Ces conflits, on cherche à les éviter par plusieurs structures plus ou moins conciliables. Socialisme, coopératisme, syndicalisme, co-gestion, entreprise privée, coexistent et forment un tout plus ou moins harmonieux. L'unité, du point de vue des travailleurs du moins, est assurée par le Histadrut, la Fédération générale des travailleurs juifs. Cette Fédération qui groupe à la fois des coopératives, des syndicats et des partis politiques, est pratiquement un état dans l'état. Elle contrôle la législation du travail, à la fois sur le plan législatif et sur le plan exécutif. De plus elle possède elle-même un bon nombre d'industries, réalisant ainsi le rêve socialiste de l'usine propriété des ouvriers.

C'est cette image complexe que monsieur Zweig essaie de nous présenter dans son ouvrage. Il réussit très bien à nous faire sentir la complexité de la situation. Mais une fois la lecture terminée, on se demande si la composition du volume ne rend pas la situation plus complexe qu'elle ne l'est en réalité. En effet on passe constamment de notions micro-sociologiques propre à la sociologie industrielle à des vues globales sur la société. Ni l'une ni l'autre de ces deux démarches n'étant assez systématiques pour donner une vue exacte des problèmes. Par ailleurs, il suppose trop de choses connues chez le lecteur. Ainsi le Histadrut est mentionné dès les premières pages mais il faut attendre à la page 238 pour en avoir une définition précise.

Malgré ces réserves sur la forme, l'ouvrage demeure très utile. Non seulement on peut en tirer des informations pertinentes sur la structure des occupations et sur les problèmes propres à certains types de travailleurs mais l'analyse du mouvement ouvrier israélien peut suggérer des réformes qui seraient utiles dans notre milieu.

GérALd Fortin. 